\title{
A sampling theorem for functions in Besov spaces on the sphere
}

\author{
Philippe Jaming \& Felipe NegreIRA \\ Univ. Bordeaux, IMB, UMR 5251 \\ F-33400 Talence, France \\ CNRS, IMB, UMR 5251 \\ F-33400 Talence, France \\ \{Philippe.Jaming, Felipe.Negreira\}@math.u-bordeaux.fr
}

\begin{abstract}
In this work we establish sampling theorems for functions in Besov spaces on the $d$-dimensional sphere $\mathbb{S}^{d}$ in the spirit of their recent counterparts established by JamingMalinnikova in [7]. The main tool is the needlet decomposition given by Narcowich et al. in [10].
\end{abstract}

\section{INTRODUCTION}

Representation and analysis of functions (signals, images or other data) on manifolds has become an ubiquitous task in many scientific fields. Indeed, high-dimensional data acquisition has become common but the meaningful data is often considered to belong to a high dimensional manifold in the ambient space. Among the most commonly encountered manifolds one can find the euclidean $d$-dimensional sphere

$$
\mathbb{S}^{d}=\left\{\left(x_{1}, \ldots, x_{d+1}\right) \in \mathbb{R}^{d+1}: x_{1}^{2}+\cdots+x_{d+1}^{2}=1\right\} .
$$

This will be the only manifold considered in this paper. Note that the dimension here refers to the dimension of the manifold, not to that of the ambient space.

Many good representing systems (orthonormal bases, frames,...) for functions on manifolds are known (see e.g. [5], [8], [10]). To properly represent a function $f$ using such a system, one usually needs to know the set of coefficients of $f$ in the representing system (or its dual) $\left\{\left\langle f, \psi_{j}\right\rangle\right\}$. However, many data acquisition systems do not provide such coefficients but rather a sample of the values of the function: $\left\{f\left(\eta_{j}\right)\right\}$.

Sampling Theory precisely deals with the possibility to reconstruct a function from its samples. The most famous result in that direction is the Shannon-Whittaker-Kotelnikov Theorem which states that a band-limited function on $\mathbb{R}$ can be reconstructed from its regular samples. This theorem has been extended in many directions e.g. one can find a version for band-limited functions on compact homogeneous manifolds (thus including the sphere) in an article of GellerPesenson [5]. The general idea of a sampling theorem can be stated as follows: if a function has moderate oscillation (for instance the function is band-limited, and thus has no highly oscillating Fourier component), then it is well approximated in the neighbourhood of a point by its value at that point. If those neighborhoods cover sufficiently well the space (the sampling rate is high enough), the sample allows to describe the function.
Recently, the first author and E. Malinnikova [7] showed that, in $\mathbb{R}^{d}$ a sampling theorem holds when moderate oscillation is measured in the scale of Besov spaces $B_{p, q}^{s}$. Our aim here is to show that a similar result holds on the sphere:

Main Theorem. Let $1 \leqslant p \leqslant \infty$. There exists finite sets of points $\left\{\eta_{1}, \ldots, \eta_{N}\right\}$ on $\mathbb{S}^{d}$, constants $c_{1}, c_{2}$ depending only on $d$, and a constant $M$ depending on $d, p$ such that, if $f \in B_{p, 1}^{d / p}\left(\mathbb{S}^{d}\right)$ with $\|f\|_{B_{p, 1}^{d / p}} \leqslant M^{-1}\|f\|_{L^{p}}$ then

$$
c_{1}\|f\|_{L^{p}} \leqslant\left(\sum_{l=1}^{N_{\varepsilon}}\left|f\left(\eta_{l}\right)\right|^{p}\right)^{1 / p} \leqslant c_{2}\|f\|_{L^{p}} .
$$

The actual result is more precise, see below. The proof consists in replacing the wavelet representation of Besov functions in $\mathbb{R}^{d}$ by the needlet representation of Besov functions in $\mathbb{S}^{d}$ as established by Narkowich-Petrushev-Ward [10] and then to adapt the computations to the spherical case.

In the next section, we introduce all material needed in the proofs so as to keep this paper self-contained. The remaining of the paper is then devoted to the proof of the main theorem.

Throughout this paper, we will adopt the following notation: on $\mathbb{R}^{d+1},|\cdot|$ and $\langle\cdot, \cdot\rangle$ are the usual Euclidean norm and scalar product while $\mathbb{S}^{d}=\left\{x \in \mathbb{R}^{d+1}:|x|=1\right\}$ is the unit sphere. We endow $\mathbb{S}^{d}$ with its usual geodesic distance $\mathrm{d}(\xi, \theta)=$ $\frac{1}{\pi} \arccos \langle\xi, \theta\rangle$ and with $\sigma$ the Lebesgue measure. The balls on $\mathbb{S}^{d}$ are denote by $B_{\eta}(r):=\left\{\xi \in \mathbb{S}^{d}: \mathrm{d}(\xi, \eta) \leqslant r\right\}$ where $\eta \in \mathbb{S}^{d}$ and $r \leq 1$.

Throughout the paper, $C$ will be a constant that changes from line to line and we add an index $C_{d}, C_{p}, C_{d, p}, \ldots$ to stress the dependence on the parameters $d, p, \ldots$. However, the constants $c^{*}, c^{* *}, c^{\diamond}, \kappa$ have specific meanings stemming from the geometry of $\mathbb{S}^{d}$ and are used to ease the reading of the arguments in the proof.

\section{PRELIMINARIES.}

In this section, we will describe a set of functions on the sphere $\mathbb{S}^{d}$ that will serve as basis for various function spaces on $\mathbb{S}^{d}$. There are two choices that might be suitable for our 
needs. One natural choice would be the wavelet type bases that can be constructed on the sphere by following e.g. the general construction for on any space of homogeneous type (see e.g. [2]). This construction is rather evolved and only leads to a frame in $L^{2}\left(\mathbb{S}^{d}\right)$. Note however that a wavelet orthonormal basis has been constructed in [1], but this is technically even more involved.

We therefore make the choice to use a simpler basis, namely the needlet system introduced by Narcowich, Petrushev and Ward in [10]. Let us now describe this system.

The starting point is the set of spherical harmonics. Recall that a spherical harmonic is an homogeneous polynomial that is harmonic (for the usual Laplace differential operator on $\left.\mathbb{R}^{d+1}\right)$. We denote by $\mathcal{H}_{\nu}$ the subspace of $L^{2}\left(\mathbb{S}^{d}\right)$ of all spherical harmonics of degree $\nu$ and by $P_{\nu}$ the orthogonal projector onto this subspace. This projection operator has an integral kernel given by a Gegenbauer polynomial, a fact that we will not need here, but allows to explicitly construct the needlet basis. We refer to [3], [12] for details. Anyway, this set the ground for our first definition.

Definition II.1. For each $N \geqslant 1$ we define the kernel $\mathcal{K}_{N}$ as

$$
K_{N}(x):=\sum_{\nu=0}^{\infty} \hat{a}\left(\frac{\nu}{N}\right) P_{\nu}(x)
$$

where $\hat{a}$ is a smooth function on $[0,+\infty)$ satisfying:

i) $\operatorname{supp} \hat{a} \subset[1 / 2,2)$,

ii) $|\hat{a}(t)|>c$ if $t \in[3 / 5,5 / 3]$,

iii) $\hat{a}^{2}(t)+\hat{a}^{2}(2 t)=1$ if $t \in[1 / 2,1]$.

Note that condition (i) of $\hat{a}$ implies that the sum in (II.1) is finite. Moreover, $\nu$ runs over $N / 2 \leqslant \nu \leqslant 2 N$ (in particular $\nu \geqslant 1$ ) and therefore $K_{N}$ is a polynomial of degree at most $2 N$. While (ii) and (iii) are not necessary for (II.1) to make sense, they are needed to form the right building blocks.

These kernels have a natural action over functions defined on $\mathbb{S}^{d}$ via the spherical convolution:

Definition II.2. Let $\Phi \in L^{\infty}[-1,1]$ and $f \in L^{p}\left(\mathbb{S}^{d}\right), 1 \leqslant$ $p \leqslant \infty$. We write

$$
\Phi * f(\xi):=\int_{\mathbb{S}^{d}} \Phi(\langle\xi, \theta\rangle) f(\theta) \mathrm{d} \sigma(\theta) .
$$

Next we define

$$
\begin{gathered}
\Phi_{0}:=P_{0} \\
\Phi_{j}:=K_{2^{j-1}} \text { for } j>0 .
\end{gathered}
$$

We then have the following result.

Proposition II.3. For each $\ell \geqslant 0$ set $\mathcal{K}_{\ell}:=\sum_{j=0}^{\ell} \Phi_{j}$. Let $1 \leqslant p \leqslant \infty$, then for every $f \in L^{p}\left(\mathbb{S}^{d}\right)$ it holds

$$
\mathcal{K}_{\ell} * f \underset{\ell \rightarrow+\infty}{\longrightarrow} f
$$

in $L^{p}\left(\mathbb{S}^{d}\right)$.

Proof. It is straightforward from the fact that $\sum_{\nu=0}^{\infty} \hat{a}\left(2^{-\nu} t\right)=1$ for all $t \in[1,+\infty)$ and the inclusion $L^{p}\left(\mathbb{S}^{d}\right) \subset \bigoplus_{\nu=0}^{\infty} \mathcal{H}_{\nu}$ for any $1 \leqslant p \leqslant \infty$.
Next, in order to define our needlet system, we need to construct a particular type of partition of $\mathbb{S}^{d}$.

Lemma II.4. For any $0<\varepsilon \leqslant 1$ there exists a partition $\mathcal{R}_{\varepsilon}=\left\{R_{1}, \ldots, R_{N_{\varepsilon}}\right\}$ of $\mathbb{S}^{d}$, together with a set of points $\mathcal{X}_{\varepsilon}=\left\{\eta_{1}, \ldots, \eta_{N_{\varepsilon}}\right\} \subset \mathbb{S}^{d}$ with the proprieties:

i) $\mathbb{S}^{d}=R_{1} \cup \cdots \cup R_{N_{\varepsilon}}$ and the sets in $\mathcal{R}_{\varepsilon}$ do not overlap $\left(\stackrel{\circ}{R}_{l} \cap \stackrel{\circ}{R}_{k}=\emptyset\right.$ if $\left.l \neq k\right)$,

ii) there exist a constant $0<c^{*}<1$ such that for every $l=1, \ldots, N_{\varepsilon}$,

$$
B_{\eta_{l}}\left(c^{*} \varepsilon\right) \subset R_{l} \subset B_{\eta_{l}}(\varepsilon) ;
$$

iii) there exist a constant $c^{* *}>0$ such that

$$
N_{\varepsilon}:=\# \mathcal{X}_{\varepsilon}=\# \mathcal{R}_{\varepsilon} \leqslant c^{* *} \varepsilon^{-d} .
$$

The constants $c^{*}$ and $c^{* *}$ depend only on the dimension $d$.

For a proof of this lemma see [9, Proposition 3.2]. It is a particular case of a more general construction of Hytönen and Kairema [6, Theorem 2.1].

Definition II.5. A set $\mathcal{X}_{\varepsilon} \subset \mathbb{S}^{d}$ which along with the associated partition $\mathcal{R}_{\varepsilon}$ of $\mathbb{S}^{d}$ has the proprieties given in Lemma II.4 will be be called a set of almost uniformly $\varepsilon$ distributed points on $\mathbb{S}^{d}$.

These sets offer a useful formula in terms of decomposition of functions.

Theorem II.6. There is a constant $c^{\diamond}$ (depending only on the dimension d) such that for any $N \geqslant 1$ and any set $\mathcal{X}_{\varepsilon} \subset \mathbb{S}^{d}$ of almost uniformly $\varepsilon$-distributed points with $\varepsilon=c^{\diamond} / N$, there exists positive coefficients $\left\{c_{\eta}\right\}_{\eta \in \mathcal{X}_{\varepsilon}}$ such that the cubature formula

$$
\int_{\mathbb{S}^{d}} p(\xi) d \xi \sim \sum_{\eta \in \mathcal{X}_{\varepsilon}} c_{\eta} p(\eta)
$$

is exact for all spherical polynomials $p(\xi)$ of degree $\leqslant N$.

In addition for all $\eta, c_{1} N^{-d} \leqslant c_{\eta} \leqslant c_{2} N^{-d}$ with the equivalence constants $c_{1}, c_{2}$ only depending on $d$.

For the construction of the needlet system, we are going to denote for each $j \geqslant 0, \varepsilon_{j}:=c^{\diamond} / 2^{j+2}, \mathcal{X}_{j}$ a fixed set of $\varepsilon_{j}$-distributed points and $\left\{c_{\eta}\right\}_{\eta \in \mathcal{X}_{j}}$ its associated family of positive coefficients. Also, we set $\mathcal{X}:=\bigcup_{j=0}^{\infty} \mathcal{X}_{j}$.

Then, from (II.2), we define our needlet system for each $j \geqslant 0$ as

$$
\varphi_{\eta}(\xi):=\sqrt{c_{\eta}} \Phi_{j}(\eta \cdot \xi), \quad \eta \in \mathcal{X}_{j} .
$$

Adding all up we have the following decomposition result.

Theorem II.7. Let $f \in L^{p}\left(\mathbb{S}^{d}\right), 1 \leqslant p \leqslant \infty$. Then

$$
f=\sum_{\eta \in \mathcal{X}}\left\langle f, \varphi_{\eta}\right\rangle \varphi_{\eta} \quad \text { in } L^{p}\left(\mathbb{S}^{d}\right) .
$$

Sketch of proof. Using the identity $f=\sum_{j=0}^{\infty} \Phi_{j} * \bar{\Phi}_{j} * f$, we decompose $\Phi_{j} * \bar{\Phi}_{j}(\xi \cdot \bullet)$ for each $j$ with Theorem II.6 and 
then we sum all over $\mathcal{X}=\bigcup_{j=0}^{\infty} \mathcal{X}_{j}$ to get (II.4). The details of the proof can be found in [10, Proposition 3.2].

The main difference that needlets have with respect to wavelets is that their localization is only measured in terms of a decay property and not by their support. More precisely, they satisfy the following size condition: for every $k \geq 0$, there exist a constant $c_{k}$ such that for every $j \geqslant 0$ and $\eta \in \mathcal{X}_{j}$ it holds

$$
\left|\varphi_{\eta}(\xi)\right| \leqslant \frac{c_{k} 2^{j d / 2}}{\left(1+2^{j} \mathrm{~d}(\xi, \eta)\right)^{k}}, \quad \forall \xi \in \mathbb{S}^{d} .
$$

And they also have a smoothness condition: there exist another constant $\kappa$ such that for every $j \geq 0$, if $\mathrm{d}(\xi, \theta) \leqslant \kappa 2^{-j}$ and $\eta \in \mathcal{X}_{j}$ then

$$
\left|\varphi_{\eta}(\xi)-\varphi_{\eta}(\theta)\right| \leqslant \frac{c_{k} 2^{j d / 2} 2^{j} \mathrm{~d}(\xi, \theta)}{\left(1+2^{j} \mathrm{~d}(\xi, \eta)\right)^{k}} .
$$

We can now define the Besov spaces in $\mathbb{S}^{d}$. Again, we follow [10].

Definition II.8. Given $0<p, q \leqslant \infty$ and $s \in \mathbb{R}$, the Besov space $B_{p, q}^{s}\left(\mathbb{S}^{d}\right)$ is defined as the set of all $f \in \mathcal{S}^{\prime}$ such that the norm

$$
\|f\|_{B_{p, q}^{s}}:=\left(\sum_{j=0}^{\infty}\left(2^{j s}\left\|\Phi_{j} * f\right\|_{L^{p}}\right)^{q}\right)^{1 / q}
$$

is finite. As usual, the $L^{p}, \ell^{q}$ norms are replaced by the supnorms when $p=\infty$ or $q=\infty$.

From this definition it is easy to see that $B_{p, q}^{s^{\prime}} \subset B_{p, q}^{s}$ when $s^{\prime} \geqslant s$, and $B_{p, q^{\prime}}^{s} \subset B_{p, q}^{s}$ when $q^{\prime} \geqslant q$. However, for our purposes, it will be more useful to characterize this space through the needlet decomposition

$$
\begin{aligned}
& \|f\|_{B_{p, q}^{s}} \\
& :=\left(\sum_{j=0}^{\infty}\left[2^{j(s+d / 2-d / p)}\left(\sum_{\eta \in \mathcal{X}_{j}}\left|\left\langle f, \varphi_{\eta}\right\rangle\right|^{p}\right)^{1 / p}\right]^{q}\right)^{1 / q}
\end{aligned}
$$

It is important to remark that these two definitions indeed coincide, for a proof of this equivalence see [10, Theorem 5.5].

\section{SAMPLING ON THE SPHERE.}

Theorem III.1. Let $1 \leqslant p \leqslant \infty, s \geq d / p, \alpha=\frac{s-d / p}{1+s-d / p}$. Fix $0<\varepsilon \leqslant 1$ and let $\left\{\zeta_{1}, \ldots, \zeta_{N_{\varepsilon}}\right\}$ be a set of almost uniformly $\varepsilon$-distributed points. Then there exists constants $C$ and $M$ depending only on $d$ and $p$, such that if $f \in B_{p, 1}^{s}\left(\mathbb{S}^{d}\right)$ with $\|f\|_{B_{p, 1}^{s}} \leqslant\left(M \varepsilon^{\alpha}\right)^{-1}\|f\|_{L^{p}}$ then

$$
\frac{1}{2 \varepsilon^{d / p}}\|f\|_{L^{p}} \leqslant\left(\sum_{l=1}^{N_{\varepsilon}}\left|f\left(\zeta_{l}\right)\right|^{p}\right)^{1 / p} \leqslant \frac{3 C}{2 \varepsilon^{d / p}}\|f\|_{L^{p}} .
$$

Proof. From (II.4) we may write $f \in B_{p, 1}^{s}\left(\mathbb{S}^{d}\right)$ as

$$
f=\sum_{j=0}^{\infty} \sum_{\eta \in \mathcal{X}_{j}}\left\langle f, \varphi_{\eta}\right\rangle \varphi_{\eta}
$$

where $\varphi_{\eta}$ are the needlets defined in (II.3). Let $\left\{R_{1}, \ldots, R_{N_{\varepsilon}}\right\}$ be the partition of $\mathbb{S}^{d}$ associated to $\left\{\zeta_{1}, \ldots, \zeta_{N_{\varepsilon}}\right\}$, take an arbitrary $l \in\left\{1, \ldots, N_{\varepsilon}\right\}$ and consider $\xi \in R_{l}$. We then have

$$
\begin{aligned}
\mid f(\xi) & -f\left(\zeta_{l}\right)\left|\leqslant \sum_{j=0}^{\infty} \sum_{\eta \in \mathcal{X}_{j}}\right|\left\langle f, \varphi_{\eta}\right\rangle|| \varphi_{\eta}(\xi)-\varphi_{\eta}\left(\zeta_{l}\right) \mid \\
& \leqslant \sum_{j=0}^{\infty}\left(\sum_{\eta \in \mathcal{X}_{j}}\left|\varphi_{\eta}(\xi)-\varphi_{\eta}\left(\zeta_{l}\right)\right|^{p^{\prime}}\right)^{1 / p^{\prime}} E_{j}(f)
\end{aligned}
$$

where for each $j \geqslant 0$

$$
E_{j}(f):=\left(\sum_{\eta \in \mathcal{X}_{j}}\left|\left\langle f, \varphi_{\eta}\right\rangle\right|^{p}\right)^{1 / p} .
$$

In order to get the right bound for (III.1), we are going to use two estimates which we will prove after showing that they imply the result.

Claim 1. For every $j \geqslant 0$ and every $\xi \in \mathbb{S}^{d}$,

$$
\left(\sum_{\eta \in \mathcal{X}_{j}}\left|\varphi_{\eta}(\xi)\right|^{p^{\prime}}\right)^{1 / p^{\prime}} \leqslant C_{p} 2^{j d / 2}
$$

where $C_{p}$ depends only on $d$ and $p$.

In particular this implies that

$$
\begin{aligned}
\left(\sum_{\eta \in \mathcal{X}_{j}}\left|\varphi_{\eta}(\xi)-\varphi_{\eta}\left(\zeta_{l}\right)\right|^{p^{\prime}}\right. & 1 / p^{\prime} \\
\leqslant 2 C_{p} 2^{j d / 2} & \leqslant C_{p} 2^{-j_{0}(s-d / p)} 2^{j(s+d / 2-d / p)}
\end{aligned}
$$

where $2^{j} \geq 2^{j_{0}}$ with $j_{0}$ to be chosen later.

Claim 2. For every $j$ such that $2^{j} \leqslant \kappa \varepsilon^{-1}$ and $\xi \in B_{\zeta_{l}}(\varepsilon)$,

$$
\left(\sum_{\eta \in \mathcal{X}_{j}}\left|\varphi_{\eta}(\xi)-\varphi_{\eta}\left(\zeta_{l}\right)\right|^{p^{\prime}}\right)^{1 / p^{\prime}} \leqslant C_{p} 2^{j d / 2} 2^{j} \mathrm{~d}\left(\xi, \zeta_{l}\right)
$$

where $C_{p}$ depends only on $d$ and $p$.

Note that since $R_{l} \subset B_{\zeta_{l}}(\varepsilon)$ by propriety (ii) in Lemma II.4, the condition $\xi \in B_{\zeta_{l}}(\varepsilon)$ is always met when $\xi \in R_{l}$.

Assuming the claims and chosing $j_{0}$ so that $2^{j_{0}} \leqslant$ $\kappa \varepsilon^{\alpha} \varepsilon^{-1} \leqslant \kappa \varepsilon^{-1}$, (III.1) reads

$$
\begin{aligned}
& \left|f(\xi)-f\left(\zeta_{l}\right)\right| \leqslant C_{p} \sum_{j=0}^{j_{0}} 2^{j d / 2} 2^{j} \mathrm{~d}\left(\xi, \zeta_{l}\right) E_{j}(f) \\
& +C_{p} 2^{-j_{0}(s-d / p)} \sum_{j=j_{0}+1}^{\infty} 2^{j(s+d / 2-d / p)} E_{j}(f) .
\end{aligned}
$$


Next, taking the $L^{p}$-norm over $R_{l}$ and using the triangular inequality, we get

$$
\begin{aligned}
& \left(\int_{R_{l}}\left|f(\xi)-f\left(\zeta_{l}\right)\right|^{p} \mathrm{~d} \sigma(\xi)\right)^{1 / p} \\
& \leqslant C_{p} \sum_{j=0}^{j_{0}} 2^{j d / 2} 2^{j}\left(\int_{R_{l}} \mathrm{~d}\left(\xi, \zeta_{l}\right)^{p} \mathrm{~d} \sigma(\xi)\right)^{1 / p} E_{j}(f) \\
& +C_{p} 2^{-j_{0}(s-d / p)} \sigma\left(R_{l}\right)^{1 / p} \sum_{j=j_{0}+1}^{\infty} 2^{j(s+d / 2-d / p)} E_{j}(f) \\
& \leqslant C_{p} \sum_{j=0}^{j_{0}} 2^{j d / 2} 2^{j} c_{d} \varepsilon^{d / p+1} E_{j}(f) \\
& +C_{p} 2^{-j_{0}(s-d / p)} \varepsilon^{d / p} \sum_{j=j_{0}+1}^{\infty} 2^{j(s+d / 2-d / p)} E_{j}(f)
\end{aligned}
$$

where we use again the fact that $R_{l} \subset B_{\eta_{l}}(\varepsilon)$. We now take $2^{j_{0}} \approx \varepsilon^{\alpha-1}$ so that $2^{-j_{0}(s-d / p)} \leqslant C \varepsilon^{\alpha}$ and thus

$$
\begin{aligned}
& \left(\int_{R_{l}}\left|f(\xi)-f\left(\zeta_{l}\right)\right|^{p} \mathrm{~d} \sigma(\xi)\right)^{1 / p} \\
& \leqslant C_{p} 2^{j_{0}} \varepsilon \varepsilon^{d / p} \sum_{j=0}^{j_{0}} 2^{j(s+d / 2-d / p)} E_{j}(f) \\
& +C_{p} 2^{-j_{0}(s-d / p)} \varepsilon^{d / p} \sum_{j=j_{0}+1}^{\infty} 2^{j(s+d / 2-d / p)} E_{j}(f) \\
& \leqslant C_{p} \varepsilon^{\alpha} \varepsilon^{d / p}\|f\|_{B_{p, 1}^{s}}
\end{aligned}
$$

which combined with the triangular inequality gives us

$$
\begin{aligned}
\left|\left(\int_{R_{l}}|f(\xi)|^{p} \mathrm{~d} \sigma(\xi)\right)^{1 / p}-\right| f\left(\zeta_{l}\right)\left|\sigma\left(R_{l}\right)^{1 / p}\right| & \\
& \leqslant C_{p} \varepsilon^{\alpha} \varepsilon^{d / p}\|f\|_{B_{p, 1}^{s}} .
\end{aligned}
$$

So, by taking $\ell^{p}$-norms and using the triangular inequality one last time, we conclude that

$$
\begin{aligned}
& \left|\|f\|_{L^{p}}-\left(\sum_{l=1}^{N_{\varepsilon}}\left|f\left(\zeta_{l}\right)\right|^{p} \sigma\left(R_{l}\right)\right)^{1 / p}\right| \\
& =\|\|\left(\int_{R_{l}}|f(\xi)|^{p} \mathrm{~d} \sigma(\xi)\right)^{1 / p} \|_{\ell_{N_{\varepsilon}}^{p}} \\
& -\left\|\left|f\left(\zeta_{l}\right)\right| \sigma\left(R_{l}\right)^{1 / p}\right\|_{\ell_{N_{\varepsilon}}^{p}} \mid \\
& \leqslant\left\|\left(\int_{R_{l}}|f(\xi)|^{p} \mathrm{~d} \sigma(\xi)\right)^{1 / p}-\left|f\left(\zeta_{l}\right)\right| \sigma\left(R_{l}\right)^{1 / p}\right\|_{\ell_{N_{\varepsilon}}^{p}} \\
& \leqslant C_{p} \varepsilon^{\alpha} \varepsilon^{d / p}\|f\|_{B_{p, 1}^{s}} N_{\varepsilon}^{1 / p} \\
& \leqslant C_{p}\left(c^{* *}\right)^{1 / p} \varepsilon^{\alpha}\|f\|_{B_{p, 1}^{s}}
\end{aligned}
$$

where $c^{* *}$ is the constant in property (iii) of Lemma II.4.
Then we have

$$
\begin{aligned}
&\left(\sum_{l=1}^{N_{\varepsilon}}\left|f\left(\zeta_{l}\right)\right|^{p} \sigma\left(R_{l}\right)\right)^{1 / p} \\
& \leqslant C_{p}\left(c^{* *}\right)^{1 / p} \varepsilon^{\alpha}\|f\|_{B_{p, 1}^{s}}+\|f\|_{L^{p}}
\end{aligned}
$$

and

$$
\begin{aligned}
&\left(\sum_{l=1}^{N_{\varepsilon}}\left|f\left(\zeta_{l}\right)\right|^{p} \sigma\left(R_{l}\right)\right)^{1 / p} \\
& \geqslant\|f\|_{L^{p}}-C_{p}\left(c^{* *}\right)^{1 / p} \varepsilon^{\alpha}\|f\|_{B_{p, 1}^{s}} .
\end{aligned}
$$

Therefore, choosing $M=2 C_{p}\left(c^{* *}\right)^{1 / p}$, we have that

$$
\frac{1}{2}\|f\|_{L^{p}} \leqslant\left(\sum_{l=1}^{N_{\varepsilon}}\left|f\left(\zeta_{l}\right)\right|^{p} \sigma\left(R_{l}\right)\right)^{1 / p} \leqslant \frac{3}{2}\|f\|_{L^{p}}
$$

whenever $\|f\|_{B_{p, 1}^{s}} \leqslant\left(M \varepsilon^{\alpha}\right)^{-1}\|f\|_{L^{p}}$. Which proves the theorem, since by the propriety (ii) in Lemma II.4 we know that $B_{\eta}\left(c^{*} \varepsilon\right) \subset R_{l} \subset B_{\eta}(\varepsilon)$ and thus $c^{*} \varepsilon^{d} \leqslant \sigma\left(R_{l}\right) \leqslant \varepsilon^{d}$.

Proof of the Claims. Let us first prove Claim 1. Using the size condition (II.5) for $\varphi_{\eta}$ with $k=d+1$ we have that

$$
\begin{aligned}
& \left(\sum_{\eta \in \mathcal{X}_{j}}\left|\varphi_{\eta}(\xi)\right|^{p^{\prime}}\right)^{1 / p^{\prime}} \\
& \leqslant c_{d+1} 2^{j d / 2}\left(\sum_{\eta \in \mathcal{X}_{j}} \frac{1}{\left(1+2^{j} \mathrm{~d}(\xi, \eta)\right)^{(d+1) p^{\prime}}}\right)^{1 / p^{\prime}} .
\end{aligned}
$$

So, in order to get the bound in Claim 1, it is enough to prove that the sum on the right hand side is bounded by a constant depending only on $d$ and $p$. To that end, define for each $\eta \in \mathcal{X}_{j}, G_{\eta}:=B_{\eta}\left(c^{*} 2^{-j}\right)$ the ball in (ii) of Lemma II.4. The triangular inequality shows that

$$
1+2^{j} \mathrm{~d}(\xi, \eta) \geqslant\left(1-c^{*}\right) \max _{\theta \in G_{\eta}}\left(1+2^{j} \mathrm{~d}(\xi, \theta)\right) .
$$

And then, as $\left\{G_{\eta}\right\}_{\eta \in \mathcal{X}_{j}}$ is a family of disjoint sets of measure $\left(c^{*}\right)^{d} 2^{-j d}$, summing over all $\mathcal{X}_{j}$ gives us

$$
\begin{aligned}
& \sum_{\eta \in \mathcal{X}_{j}} \frac{1}{\left(1+2^{j} \mathrm{~d}(\xi, \eta)\right)^{(d+1) p^{\prime}}} \\
& \leqslant \sum_{\eta \in \mathcal{X}_{j}} \frac{\left(1-c^{*}\right)^{-(d+1) p^{\prime}}}{\sigma\left(G_{\eta}\right)} \int_{G_{\eta}} \frac{1}{\left(1+2^{j} \mathrm{~d}(\xi, \theta)\right)^{(d+1) p^{\prime}}} \mathrm{d} \sigma(\theta) \\
& \leqslant\left(1-c^{*}\right)^{-(d+1) p^{\prime}} \int_{\mathbb{S}^{d}} \frac{2^{j d}}{\left(1+2^{j} \mathrm{~d}(\xi, \theta)\right)^{(d+1) p^{\prime}}} \mathrm{d} \sigma(\theta) \\
& \leqslant c_{p}\left(1-c^{*}\right)^{-(d+1) p^{\prime}}
\end{aligned}
$$

by standard calculus. This completes the proof of Claim 1 . The proof of Claim 2 is exactly the same using (II.6) instead of (II.5) in (III.2). 
As the needlets $\varphi_{\eta}$ are continuous functions (moreover they are polynomials) the same computations above and the fact the $\mathbb{S}^{d}$ is compact show that every $f \in B_{p, 1}^{s}\left(\mathbb{S}^{d}\right)$ is continuous.

Also, another corollary that can be drawn from the previous calculations is the following result:

Corollary III.2. Let $1 \leqslant p \leqslant \infty, s \geq d / p$ and $\alpha=\frac{s-d / p}{1+s-d / p}$. Fix $\varepsilon>0$ and let $\left\{\zeta_{1}, \ldots, \zeta_{N_{\varepsilon}}\right\}$ be a set of almost uniformly $\varepsilon$-distributed points and let $\left\{R_{1}, \ldots, R_{N_{\varepsilon}}\right\}$ be the associated partition of $\mathbb{S}^{d}$. Then there exists a constant $C_{p}$ depending only on $d$ and $p$ such that

$$
\int_{\mathbb{S}^{d}}\left|f(\xi)-\sum_{l=1}^{N_{\varepsilon}} f\left(\zeta_{l}\right) \mathbf{1}_{R_{l}}(\xi)\right|^{p} \mathrm{~d} \sigma(\xi) \leqslant C_{p} \varepsilon^{\alpha}\|f\|_{B_{p, 1}^{s}}
$$

holds true for all $f \in B_{p, 1}^{s}\left(\mathbb{S}^{d}\right)$.

\section{CONCLUSION AND FUTURE WORK}

In this paper, we have used the needlet decomposition of Besov spaces to establish a sampling theorem in $B_{p, 1}^{s}$ when the smootheness index is large enough. This opens the path to future work in two directions:

- establish efficient algorithms for reconstructions of function on a sphere from their samples;

- extend this work to more general compact Riemannian manifolds for which needlet like decompositions have been established in [5], [8] and then to more general homogeneous spaces using wavelet decompositions like those given in [2]. Note that some results in this direction have already been obtained by various authors. For instance $\mathrm{H}$. Führ and K. Gröchenig [4] obtained sampling theorems under oscillation conditions on locally compact groups while I.Z. Pesenson [11] established some links between sampling and smallness of Besov norms on compact Riemannian manifolds.

\section{REFERENCES}

[1] P. Auscher \& T. Hytonen Orthonormal bases of regular wavelets in spaces of homogeneous type. Applied and Computational Harmonic Analysis 34 (2013), 266-296.

[2] D. Deng \& Y. Han, Harmonic Analysis on Spaces of Homogeneous Type. Springer Lecture Notes in Math 1966, 2009, Springer, New York.

[3] F. DAI \& Y. XU, Approximation Theory and Harmonic Analysis on Spheres and Balls. Springer Monographs in Math, 2013, Springer, New York.

[4] H FÜHR \& K GRÖCHENIG, Sampling theorems on locally compact groups from oscillation estimates. Mathematische Zeitschrift 255 (2007), 177-194.

[5] D. Geller \& I. Pesenson, Band-limited localized Parseval frames and Besov spaces on compact homogeneous manifolds. Journal of Geometric Analysis 21 (2011), 334-371.

[6] T. Hytönen \& A. KAIREMA, Systems of dyadic cubes in a doubling metric space. Colloquium Mathematicum 126 (2012), 1-33.

[7] Ph. Jaming \& E. Malinnikova, An uncertainty principle and sampling inequalities in Besov spaces. Journal of Fourier Analysis and Applications 22 (2016), 768-786.

[8] M. Maggioni \& H. N. Mhaskar, Diffusion Polynomial Frames on measure metric spaces. Applied and Computational Harmonic Analysis 24 (2007), 329-353.

[9] H. Mhaskar, F. NARCowich \& J. Ward, Spherical MarcinkiewiczZygmund inequalities and positive quadrature. Journal of Mathematics of Computation 70 (2001), 1113-1130.

[10] F. Narcowich, P. Petrushev \& J. Ward, Decomposition of Besov and Triebel-Lizorkin spaces on the sphere. Journal of Functional Analysis 238 (2006), 530-64.
[11] I.Z. Pesenson, Paley-Wiener approximations and multiscale approximations in Sobolev and Besov spaces on manifolds. J. Geom. Anal. 4 (2009), 101-121.

[12] E.M. STEIN \& G. Weiss, Introduction to Fourier Analysis on Euclidean spaces. Princeton University Press, 1971. 